\title{
Carbene-induced rescue of catalytic activity in deactivated Nitrite Reductase mutant
}

Matteo Planchestainer, ${ }^{[a, b]}$ Jonathan McMaster, ${ }^{[b]}$ Christine Schulz, ${ }^{[c]}$ Francesca Paradisi*[a,b] and Martin Albrecht*[a]

[a] University of Bern, Department of Chemistry \& Biochemistry, Freiestr. 3, 3012 Bern, Switzerland

${ }^{[b]}$ University of Nottingham, School of Chemistry, University Park, Nottingham NG7 2RD, UK

${ }^{[c]}$ Max-Planck Institut für Kohlenforschung, Department of Molecular Theory and Spectroscopy, KaiserWilhelm-Platz 1, 45470 Mülheim an der Ruhr, Germany

$\begin{array}{lll}\text { Correspondence: } & \text { francesca.paradisi@dcb.unibe.ch } & \text { Twitter: @Paradisi_Lab } \\ & \text { martin.albrecht@dcb.unibe.ch } & \text { Twitter: @Albrecht_Lab }\end{array}$

\begin{abstract}
The role of His145 in the T1 copper center of Nitrite Reductase (NiR) is pivotal for the activity of the enzyme. Mutation to a glycine at this position enables the reconstitution of the T1 center by the addition of imidazole as exogenous ligands, however the catalytic activity is only marginally rescued. Here we demonstrate that the uptake of 1,3-dimethylimidazolylidene as $\mathrm{N}$-heterocyclic carbene (NHC) by the H145G NiR mutant instead of imidazole yields a significantly more active catalyst, suggesting a beneficial role of such C-bonding. Spectroscopic analyses of the formed H145G NHC variant as well as an analogue without the catalytic T2 copper center reveals no significant alteration of the T1 site compared to the wild type or the variant containing imidazole as exogenous $\mathrm{N}$-bound surrogate of $\mathrm{H} 145$. However, the presence of the carbene doubles the catalytic activity of the mutant compared to the imidazole variant. This enhanced activity has been attributed to a faster electron transfer to the $\mathrm{T} 1$ center in the NHC variant and a concomitant change of the rate-limiting step.
\end{abstract}




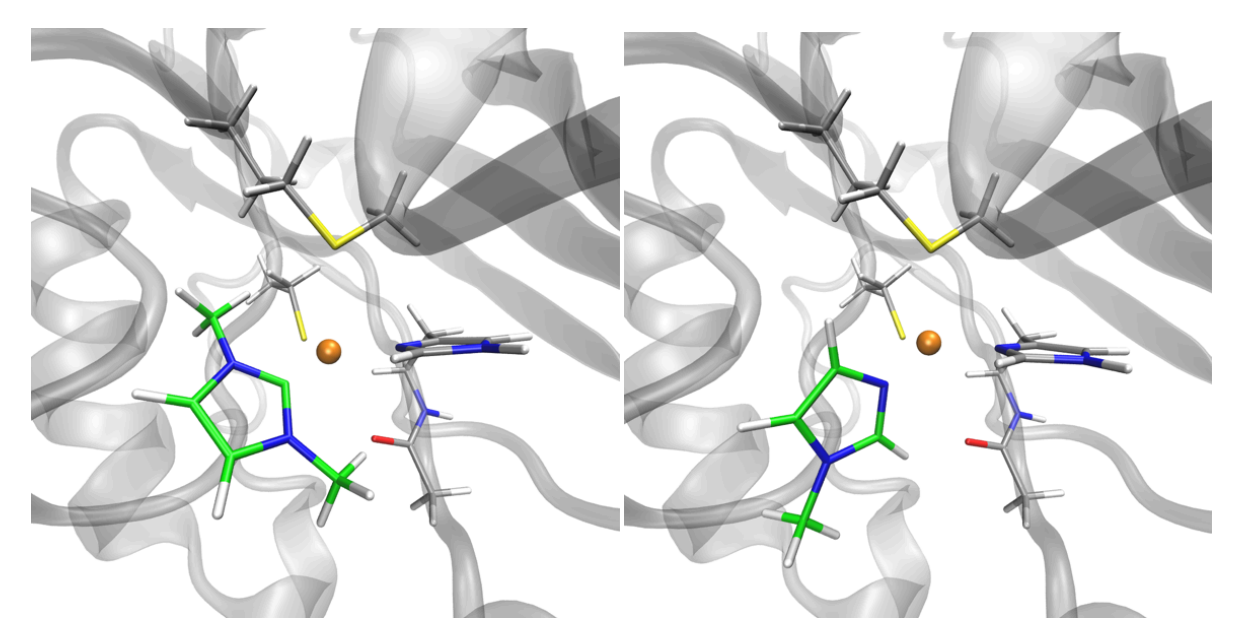

\section{Introduction}

Carbenes, a two valence electron-neutral carbon species, and in particular N-heterocyclic carbenes (NHCs), have been key moieties employed in the design of metal-based catalysts, which allow for the modulation of the activity and selectivity of the metal center ${ }^{[1-3]}$ Despite the compelling properties imparted by the carbon-coordination mode, examples of such species in biologicallyrelevant systems are rare, ${ }^{[4]}$ and no naturally occurring carbenes have been observed as coordinating ligands in metalloproteins. The only examples of NHC-metal coordination in biologically-relevant environments have been imposed by combining synthetic catalysts with protein scaffolds to generate hybrid enzymes, which exploit the promiscuous activity provided by the metal center and the regioand/or stereo-selective properties imparted by the peptide structure..$^{[5-7]}$ Very recently, however, carbene-metal binding was observed in fully natural enzymes as intermediate step in the enzymatic $\mathrm{C}-\mathrm{H}$ functionalization catalyzed by iron hemoproteins. ${ }^{[8,9]}$

In this context, we exploited a small copper protein, the Azurin from $P$. aeruginosa, to demonstrate that carbene coordination facilitates reduction processes at the $\mathrm{Cu}$ center. The solvent 
exposed coordinating histidine of the T1 Cu-center was substituted with an exogenous NHC ligand, which successfully restores the spectroscopic properties and decreases the redox potential. ${ }^{[10]}$ Furthermore, we speculated that the tautomerization of imidazole to NHC occurring in the histidine ring might act as switch to modulate the catalytic events in some metallo-enzymes. Histidine is indeed a common amino acid often exploited as metal coordination moiety at the active site of enzymes and binds to a metal center through the nitrogen of its imidazole ring. ${ }^{[11,12]}$ In hemoproteins, for example, the axial binding of histidine to the heme prosthetic group controls the reactivity of the metal center and triggers the binding and release of small molecules, such as $\mathrm{O}_{2}$, in the trans position. ${ }^{[13]}$ Generally histidine is considered to bind through a side chain nitrogen to the metal center, and this bonding mode has been established through countless spectroscopic and crystallographic studies. ${ }^{[14]}$ The alternate carbon-coordination offers a rationale for those electron transfer and catalytic processes that occur at low redox potentials. Therefore, we expanded our approach to a catalytically active Cuenzyme to probe the influence of a NHC ligand on the enzymatic properties.

Cu-containing proteins mediate numerous electron transfer processes, yet they operate over a broader redox range than their standard reduction potentials would suggest. ${ }^{[14-16]}$ Azurin (Azu) and pseudo-azurin typically act as an electron shuttle to redox enzymes, such as nitrite reductase (NiR), which is found in the dissimilatory denitrification pathways of certain Alcaligenes $s p$. (A. faecalis and A. xylosoxidans), and Psudomonas genus among others. ${ }^{[17]}$ Nitrite reductases possess two Cu centers in entatic states, ${ }^{[18,19]}$ a T1 center that possesses a similar coordination sphere to the Cu center in Azu, and a type 2 (T2) center, buried in the enzyme structure where the reduction of $\mathrm{NO}_{2}^{-}$to $\mathrm{NO}^{-}$takes place (Fig 1A). In vivo, electrons are shuttled from the Azu-T1 to the NiR-T1 in a transient proteinprotein complex, and then from NiR-T1 to T2 where the substrate $\mathrm{NO}_{2}^{-}$is eventually reduced to NO. ${ }^{[20-22]}$ In the NiR from A. faecalis, mutations of the His145 ligand at the T1 Cu center result in variants that behave similarly to Azu-H117G. The His145 residue bridges the solvent-accessible area to the Cu center and allows for the coordination of an exogenous ligand to this T1 Cu site. In Azu, the 
reconstitution is limited to the restoration of the UV/vis and EPR spectroscopic fingerprint, yet the lack of catalytic properties does not allow any further considerations. ${ }^{[23]}$

In this work, we have prepared the H145G NiR mutant and introduced an NHC precursor to investigate the implications of a C-bound ligand at a T1 Cu-center of an active metallo-enzyme. ${ }^{[24]}$ The enhanced activity of this NHC system compared to the imidazole-rescued analogue underpins the potential of these C-coordinated ligands in redox processes mediated by metallo-enzymes. Moreover, computational studies provided also insights on the stability of such a C-bonding mode of the imidazole heterocycle.
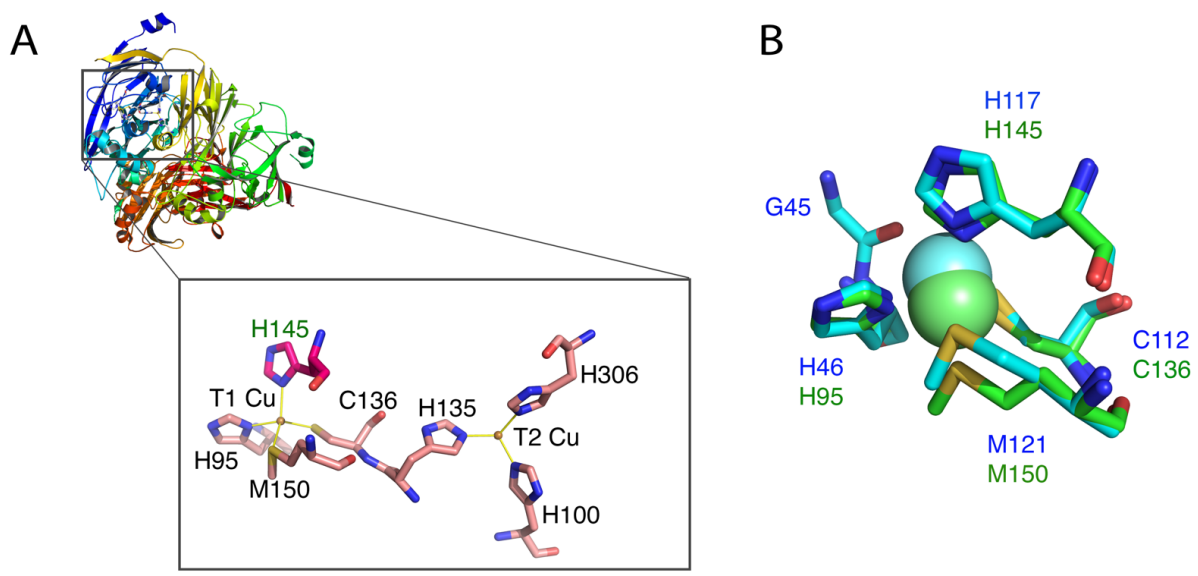

Figure 1. A) Schematic representations of nitrite reductase (from A. faecalis - PDB 2FJS) and close up of the Cu binding sites, including the T1 Cu site involved in electron transfer and bound by the same set of amino acids as azurin, and the catalytic T2 Cu site bound by three histidine ligands and engaged in the actual reduction of nitrite to nitric oxide. ${ }^{[25,26]}$ B) Overlay of T1-Cu sites of the nitrite reductase (green) and the one of the small copper protein Azurin (from P. aeruginosa - PDB: 3U25, cyan).

\section{Results and Discussion}

\section{Protein preparation and spectroscopic features}

Clones of the wild type (WT) and H145G variants of NiR harbored in a pET28a vector ${ }^{[26]}$ were used as templates to generate a cleavable poly-His-tag version of both proteins through the introduction of a Tobacco Etch Virus (TEV) endopeptidase cleavage sequence. This approach led to an easy removal of the poly-histidine tail used for purification to avoid potential interference through coordination to $\mathrm{Cu}(\mathrm{II})$ ions. Samples of the WT and H145G mutant NiR were purified by single step metal affinity 
chromatography (Figure S1), followed by poly-his tag cleavage using 50:1 TEV proteases, and buffer exchange by gel-filtration. Cleavage and structural integrity were verified by mass spectrometry (Figure S2). Due to the superimposition of the T1 and T2 signals in EPR spectroscopy, the catalytic T2 copper center in NiR was silenced by modification of the bonding site through a H306A mutation, which is known to suppress copper binding to this site. ${ }^{[27]}$ The NiR T2 mutants (H306A and H145GH306A) were successfully prepared following the same procedure (Figure S1, S2).

$\mathrm{Cu}(\mathrm{II})$ binding of the mutant in the presence of imidazole ( $\mathrm{H} 145 \mathrm{G}$ Imid 3, Figure 2) was verified by UV-vis (Figure 3A) and EPR spectroscopy (Figure S11). Frozen solution X-band EPR spectroscopy at $77 \mathrm{~K}$ revealed the classic signature of a reconstituted T1 Cu center $\left(\mathrm{g}_{\|}=2.196, \mathrm{~A}_{\|}=76 \times 10^{-4} \mathrm{~cm}^{-1}\right.$, Table 1) superimposed with the unaltered resonance pattern of the internal T2 center $\left(g_{\|}=2.341, A_{\|}\right.$ $\left.=156 \times 10^{-4} \mathrm{~cm}^{-1}\right)$. These observations are in excellent agreement with literature data and confirm that the H145G mutation does not significantly affect the structure of the protein and that the spectroscopic signatures can be restored upon coordination of imidazole (Imid). ${ }^{[28-30]}$

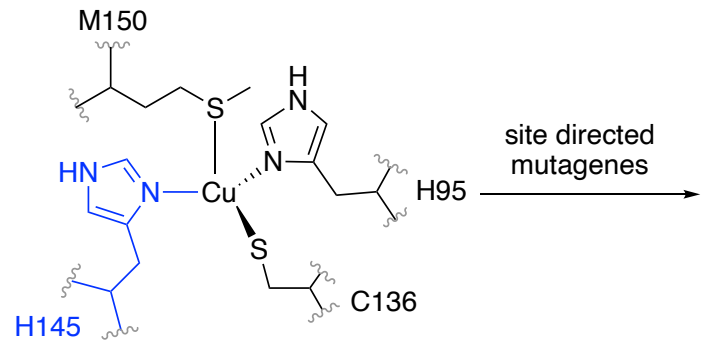

NiR WT (1)

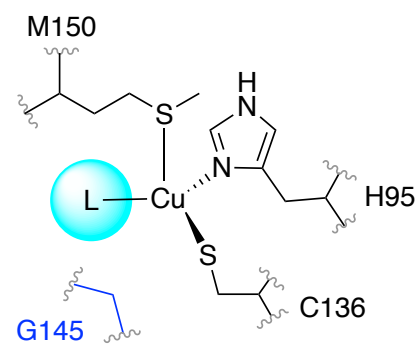

NiR H145G L

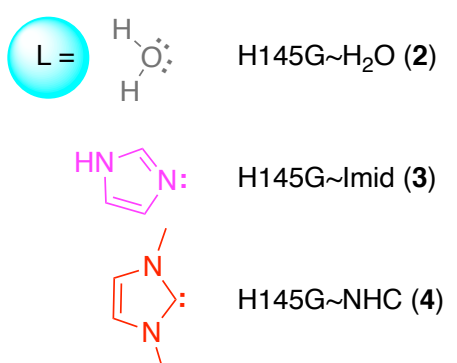

Figure 2. Schematic representation of the T1 site of NiR WT (1), and the mutant H145G containing water (2), an imidazole (3), and a NHC (4) ligand as analogues of the $\mathrm{N}$ - and $\mathrm{C}$-bonding modes of His, respectively.

Following our previously established method, ${ }^{[10]}$ dimethylimidazolium carboxylate $\left(\mathrm{NHC}-\mathrm{CO}_{2}\right)$ was used as precursor to install an exogenous carbene ligand at the T1 Cu center of NiR H145G (H145G NHC 4, Figure 2) by decarboxylation. An excess of $N, N^{\prime}$-dimethylimidazolium carboxylate (250 
equiv) was used in the presence of the $\mathrm{H} 145 \mathrm{G} \sim \mathrm{H}_{2} \mathrm{O}$ mutant 2 in aqueous MES buffer $\mathrm{pH}$ 7. The addition of the carbene precursor induced a rapid color change of the protein in solution from a very light grey to intense green/grey color, identical to the change observed when reconstituting the T1 Cu center of the $\mathrm{H} 145 \mathrm{G}^{\sim} \mathrm{H}_{2} \mathrm{O}$ mutant with exogenous Imid (Figure 3B). The UV-vis spectra of the WT and the H145G Imid and H145G NHC mutants are similar and feature three distinct absorption maxima at $c a$. 390, 460, and $590 \mathrm{~nm}\left(\varepsilon 1.5-3 \mathrm{mM}^{-1} \mathrm{~cm}^{-1}\right.$; Table 2, Figure 3). The maxima shift by less than $7 \mathrm{~nm}$ upon variation of exogeneous ligand, though the exact maxima are difficult to determine accurately due to the broadness of the absorption bands. In the absence of either NHC or Imid as exogenous ligand, H145G H2O features two T2 copper centers with radically altered electronic spectra (three very broad and weak absorptions at 360,450, and $600 \mathrm{~nm}$, with extinction coefficients $<1.0 \mathrm{mM}^{-1} \mathrm{~cm}^{-1}$ ), providing a diagnostic tool to monitor Imid and NHC bonding to the mutant. It is notable that neither the NHC nor the Imid ligand fully reconstitute the T1 center. Reconstitution is estimated at $50 \%$ and $75 \%$, respectively, based on the extinction coefficient at $\lambda_{\max }$ around $590 \mathrm{~nm}$. Remarkably, the T2 mutation (H306A) did not alter the UV-vis signature of the parent NiR construct, nor of the double mutations with a $\mathrm{H} 145 \mathrm{G}$ site, irrespective of the exogenous ligand $\left(\mathrm{H}_{2} \mathrm{O}\right.$, Imid, NHC; Table 2, Figure S7, S8). 

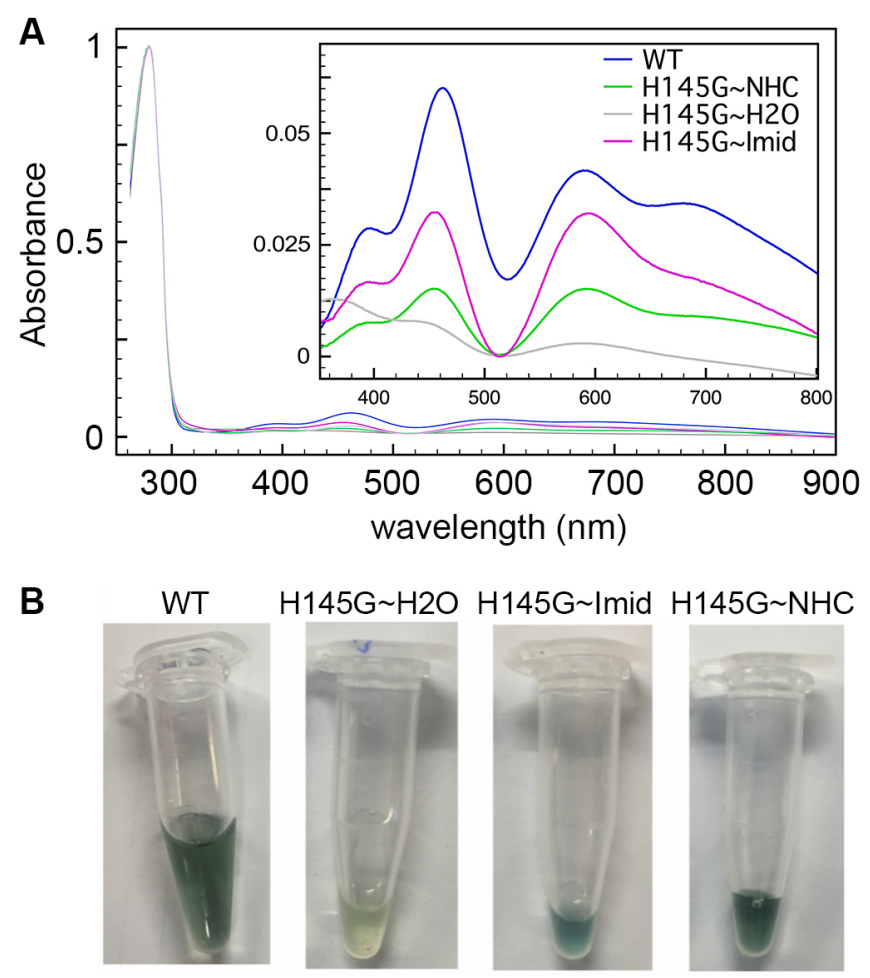

Figure 3. A) UV-vis spectra of the wild-type, and the $\mathrm{H} 145 \mathrm{G}$ mutant without any ligand $\left(\mathrm{H}_{2} \mathrm{O} ; 2\right)$, with imidazole (Imid; 3 ) or $N, N^{\prime}$-dimethylimidazolylidene (NHC; 4) coordinated to the copper site. The UV-vis spectra show the essentially identical absorption characteristics $\left(\lambda_{\max }\right)$ of NiR WT and the H145G mutants restored with Imid or NHC. Spectra of NiR WT, NiRH145G $\sim \mathrm{H}_{2} \mathrm{O}$ and NiRH145G Imid have been recorded for reference and fully agree with reported data. ${ }^{[23]}$ The insets shows a expansion of the visible region in the $0-3 \mathrm{mM}^{-1} \mathrm{~cm}^{-1}$ extinction coefficient range. B) Samples of NiR WT and H145G showing the diagnostic color of the variants upon addition of exogenous ligand.

Table 1. Spectroscopic data for NiR wild-type and H145G and H306A mutants containing Imid and NHC as exogenous ligands ${ }^{\text {a) }}$

\begin{tabular}{|c|c|c|c|c|c|c|}
\hline & \\
\hline & $\begin{array}{l}\lambda_{\max } \\
(\mathrm{nm})\end{array}$ & $\begin{array}{c}\varepsilon_{\mathrm{app}}^{\mathrm{b})} \\
\left(\mathrm{mM}^{-1} \mathrm{~cm}^{-1}\right)\end{array}$ & & $\mathrm{g}_{\perp}$ & $\mathrm{g}_{\|}$ & $\begin{array}{c}\mathrm{A}_{\|} \\
\left(10^{-4} \mathrm{~cm}^{-1}\right)\end{array}$ \\
\hline \multirow[t]{3}{*}{ WT (1) } & 392 & 1.63 & $\mathrm{~T} 1$ & 2.069 & 2.185 & 80 \\
\hline & 462 & 2.90 & $\mathrm{~T} 2$ & 2.056 & 2.341 & 156 \\
\hline & 589 & 2.17 & & & & \\
\hline \multirow[t]{3}{*}{$\mathrm{H} 145 \mathrm{G} \sim \mathrm{H}_{2} \mathrm{O}(2)$} & 367 & 1.02 & $\mathrm{~T} 1$ & 2.069 & 2.333 & 125 \\
\hline & 444 & 0.81 & $\mathrm{~T} 2$ & 2.054 & 2.346 & 146 \\
\hline & 591 & 0.62 & & & & \\
\hline \multirow[t]{3}{*}{ H145G Imid (3) } & 395 & 1.15 & $\mathrm{~T} 1$ & 2.060 & 2.196 & 76 \\
\hline & 457 & 1.80 & $\mathrm{~T} 2$ & 2.070 & 2.340 & 140 \\
\hline & 593 & 1.78 & & & & \\
\hline \multirow[t]{3}{*}{ H145G NHC (4) } & 394 & 0.81 & $\mathrm{~T} 1$ & 2.035 & 2.193 & 83 \\
\hline & 455 & 1.11 & $\mathrm{~T} 2$ & 2.070 & 2.350 & 170 \\
\hline & 592 & 1.11 & & & & \\
\hline \multirow[t]{3}{*}{ H306A } & 398 & 1.46 & $\mathrm{~T} 1$ & 2.054 & 2.191 & 70 \\
\hline & 462 & 2.39 & & & & \\
\hline & 589 & 1.85 & & & & \\
\hline
\end{tabular}




$\begin{array}{lllllll}\text { H306A-H145G H2O } & 370 & 1.02 & \text { T1 } & 2.058 & 2.278 & 181 \\ & 445 & 0.63 & & & & \\ \text { H306A-H145G Imid } & 639 & 0.69 & & & & \\ & 391 & 0.92 & \text { T1 } & 2.050 & 2.194 & 68 \\ \text { H306A-H145G NHC } & 461 & 1.37 & & & & \\ & 593 & 1.63 & & & & \\ & 374 & 1.09 & \text { T1 } & 2.060 & & \\ & 454 & 1.11 & & & & \\ & 589 & 1.12 & & & & \end{array}$

\footnotetext{
a) UV-vis spectra in MES buffer (10 mM MES, pH 7.0); X-band EPR spectra of frozen $0.5 \mathrm{mM}$ protein solution in glycerol:MES buffer $\mathrm{pH} 7(50: 50 \mathrm{v} / \mathrm{v} \%)$ at $77 \mathrm{~K}$ with a modulation amplitude of $5 \mathrm{G}$; ${ }^{\text {b) }}$ apparent extinction coefficient of the solution $\varepsilon_{\mathrm{app}}$, calibrated to the molar extinction coefficient at $280 \mathrm{~nm}$ of NiR $\left(\varepsilon_{280}=46 \mathrm{mM}^{-1} \mathrm{~cm}^{-1}\right.$ [23]).
}

$\mathrm{N}$ - and C-bonding of the exogenous ligands in the H145G mutant was further investigated by EPR spectroscopy. While in all constructs, the buried T2 Cu site remains essentially unchanged (Table 1 , Figure S11), the signature of the peripheral $\mathrm{Cu}$ center is diagnostic. A distinct shift is observed between the WT and the $\mathrm{H} 145 \mathrm{G} \sim \mathrm{H}_{2} \mathrm{O}$ holo mutant, as the latter reveals a large hyperfine splitting indicative of a T2 Cu site. The binding of a carbene unit to the Cu center in this H145G mutant results in EPR spectroscopic data very similar to those of the WT and of the H145G Imid mutant (Table 1, Figure S11), suggesting successful restoration of a T1 site. As observed in the UV traces, the EPR spectra confirm with remarkable accuracy the same level of reconstitution (50\% for NHC, $69 \%$ for Imid). The EPR data are reminiscent of those observed for the azurin mutants, which is expected when considering the essentially identical structure and electronic configuration of the T1 site in both enzymes. The EPR spectra for the T2 mutant (H306A) could be simulated to unambiguously extract the g values and hyperfine splitting of the peripheral T1 copper center for all mutants (Table 1, Figure 4). 


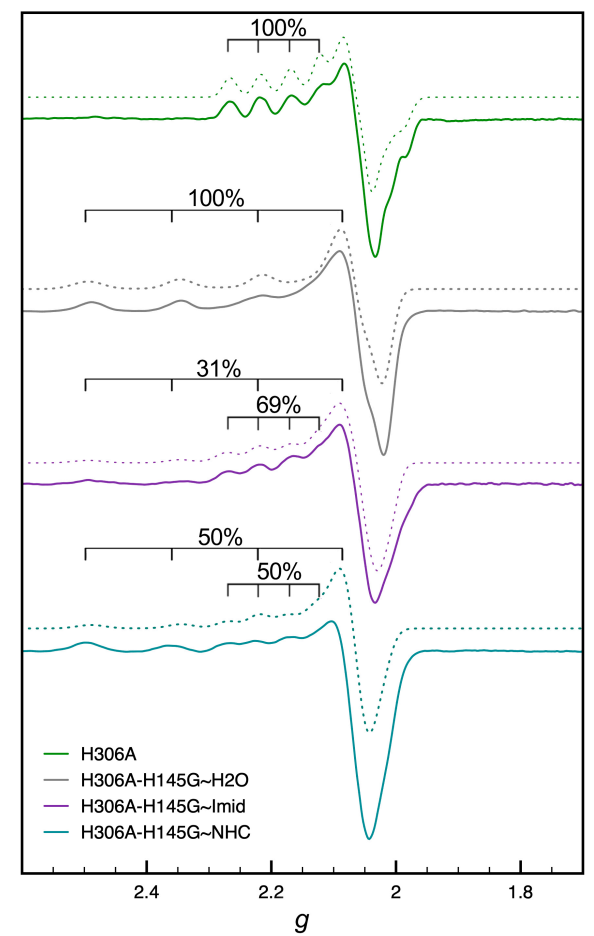

Figure 4. Stacked EPR spectra of NiR H306A and H306A-H145G mutants. H306A mutant (green trace) showing the clear T1 features, H145G-H306A mutant without any ligand $\left(\mathrm{H}_{2} \mathrm{O}\right)$ and containing an imidazole ligand (Imid, 10 eq.) and an Nheterocyclic carbene ligand (NHC, 10 eq.). Spectra of NiR H306A have been recorded for reference and fully agree with reported data. ${ }^{[27]}$ Simulated spectra shown as dashed lines.

To exclude alternative binding modes of the carboxylate-NHC precursor, a series of control ligands were investigated by UV-vis and EPR spectroscopy. These control ligands included i) 1,3dimethylimidazolium tetrafluoroborate $(\mathrm{dMI})$ and 1,2,3-trimethyl-imidazolium tetrafluoroborate (tMI) to exclude the formation of alternative carbene cordination modes, such as abnormal C4-bound carbenes or $\mathrm{Cu}-\pi$ interactions; ${ }^{[31]}$ ii) sodium benzoate (Benz) to mimic the carboxylate binding that would be expected if decarboxylation in the $\mathrm{NHC}-\mathrm{CO}_{2}$ precursor were inhibited (Figure S4, S9, S12; Table S2, S3); and iii) 1,2-dimethylimidazole (DMI) to probe the unexpected migration of a methyl group from nitrogen to the carbenic carbon. None of these control ligands was able to reproduce the WT features, supporting the NHC coordination mode to the T1 Cu-center in H145G NHC. Of note, while DMI-binding to the H145G mutant was not detectable (Figure S5, S10; Table S2, S3), DMI showed a normal nitrogen binding behavior with the Azurin, ${ }^{[10]}$ (Figure S16), with a coordination stability comparable to $\mathrm{N}$-methyl imidazole (NMI; Figure S17). This could be due to the fact that while the NiR 
and Azu T1 copper sites can be confidently overlaid, the NiR T1 site is buried deeper in the protein structure than in Azu.

Interestingly, the UV-vis signature of $\mathrm{H} 145 \mathrm{G} \sim \mathrm{NHC}$ was stable for the first two hours after sample preparation (Figure S6), but it gradually lost intensity and bleaching was complete $\left(\varepsilon<0.4 \mathrm{mM}^{-1} \mathrm{~cm}^{-1}\right)$ within $48 \mathrm{~h}$. This bleaching may be rationalized either by stripping of the Cu-NHC unit or with a reduction of the $\mathrm{Cu}(\mathrm{II})$ center to $\mathrm{Cu}(\mathrm{I})$ in the T1 site. To better understand this behavior, we replicated the bleaching conditions with Azurin, ${ }^{[10]}$ which features essentially the same T1 Cu coordination (cf Fig. 1B), yet it has a much simpler UV-vis signature to monitor changes over time. Azurin was investigated under a range of conditions. The addition of fresh $\mathrm{Cu}(\mathrm{II})$ or $\mathrm{Cu}(\mathrm{II})$ and $\mathrm{NMI}$ to an aged and bleached sample (72 h) of AzuH117G NHC did not rescue the UV-vis signature (Figure S13, S14), suggesting that the T1 site is either compromised $^{[32,33]}$ or still occupied. Therefore, a $72 \mathrm{~h}$ aged and bleached sample of AzuH117G NHC was washed repeatedly with buffer to remove ions and labile ligands (see SI). The sample was then treated as if freshly purified, i.e. incubated with both $\mathrm{Cu}(\mathrm{II})$ and NMI. This procedure recovered the pertinent UV/vis features $\left(\lambda_{\max }=630 \mathrm{~nm}\right)$ and up to $77 \%$ of its original absorbance $\left(\varepsilon_{72 \mathrm{~h}}=3.4 \mathrm{mM}^{-1} \mathrm{~cm}^{-1}\right.$ vs $\varepsilon_{\text {fresh }}=4.4 \mathrm{mM}^{-}$ $\left.{ }^{1} \mathrm{~cm}^{-1}\right),{ }^{[10]}$ indicating that the T1 site was almost completely accessible (Figure S15). The copper present in the samples and washing fractions was quantified by inductively coupled plasma mass spectrometry (ICPMS), which confirmed that the washed protein retained $\sim 35 \%$ of the metal with respect to the unwashed sample. This partial retention explains why the washed sample did not regain the full intensity at $630 \mathrm{~nm}$. These data suggest that the bleaching effect is due to $\mathrm{Cu}(\mathrm{II})$ to $\mathrm{Cu}(\mathrm{I})$ reduction promoted by the NHC binding and not due to alteration of the amino acid side chains of the $\mathrm{T} 1$ coordination site (e.g. cysteine oxidation). Further support for such a reduction was obtained from comparative EPR analyses of freshly prepared and aged samples of AzuH117G NHC as well as the NiR analogue, which indicate the disappearance of the characteristic T1 signature, yet no formation of a T2 center or resonances due to dissolved $\mathrm{Cu}(\mathrm{II})$ ions were observed (Figure S18). $\mathrm{Cu}(\mathrm{I})$ binding to the T1 site is expected to be weaker than 
$\mathrm{Cu}(\mathrm{II})$ binding, as indicated by the $\mathrm{Cu}$ content detected in the washing fractions (Table S6). In contrast to the C-bonding of $\mathrm{NHC}$, analogous $\mathrm{N}$-binding of an exogenous ligand by coordination of $\mathrm{NMI}$ to the AzuH117G copper mutant resulted in a sample that showed a much higher stability, losing only $20 \%$ of its intensity within the first $48 \mathrm{~h}$ (Figure S17). These findings lend strong support to the fact that NHC preferentially stabilizes low-valent $\mathrm{Cu}(\mathrm{I})$ centers while the $\mathrm{N}$-ligand favors high-valent $\mathrm{Cu}(\mathrm{II})$ species. ${ }^{[34]}$ Efforts to further probe this ligand impact by electrochemistry were unsuccessful, and despite numerous approaches (microelectrode, electrode coating), no reliable redox signal could be recorded. ${ }^{[35]}$

Carbene bonding to the T1 copper site has been further probed theoretically using both the smaller Azu as well as NiR. In a first stage, small quantum chemical models for the Cu site of Azu were used, consisting of the residues Cys112, Met121 and His46 as well as the backbone carbonyl of Gly45, which acts as the fifth ligand to $\mathrm{Cu}$. To validate the model, the original Azu active site containing His117 was considered as well. For the external ligands, an unsubstituted imidazole and an unsubstituted NHC were used to optimize two tautomers. In a second step, the effective ligands (NMI and NHC, respectively) were used to evaluate the stereochemical influence of the methyl groups on the binding modes. The construction of the protein model assumes that the H117G mutant follows the same folding pattern and maintains the Cu site relatively intact, as demonstrated experimentally. The calculated model is validated by the comparison of geometries and the correct reproduction of the ligand hyperfine coupling constants of the Azu WT within the limits of DFT (Table S7-S8, Figure S19). In silico introduction of the NHC induces only minimal geometrical changes and the T1 center is fully preserved. Remarkably, the NHC system is computed to have about the same energy as the NMI analogue. While the Azurin is a somewhat simplified model, the same analysis performed on the T1 NiR yielded virtually identical results (Table S9-S10, Figure S20). In fact, due to the distortion of the T1 site, the differences among the non-native ligands (NHC and Imi) are even smaller in NiR than in Azu. 


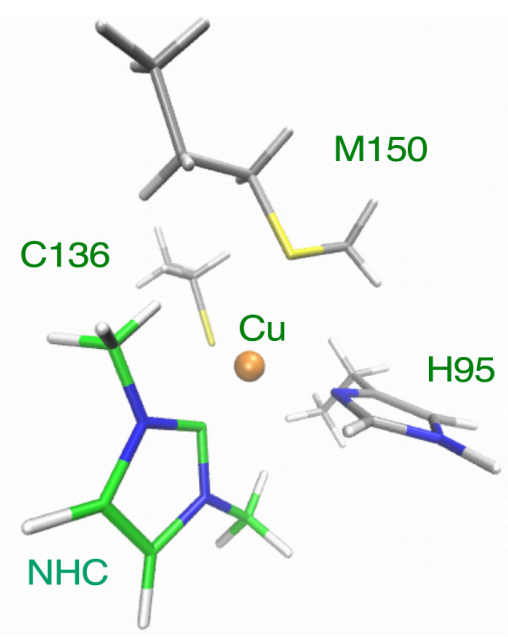

Figure 5. Optimized QM model for the T1 site of the NiR H145G NHC mutant.

\section{Enzymatic activity}

The catalytic relevance of carbene bonding in the NiR instead of a histidine in the WT was probed by monitoring the enzymatic reduction of nitrite $\left(\mathrm{NO}_{2}^{-}\right)$to nitric oxide (NO). ${ }^{[20,23]}$ Activity assays using methyl-viologen as sacrificial reducing agent showed a WT activity of $899 \mathrm{U} \mathrm{mg}^{-1}$, while the $\mathrm{H} 145 \mathrm{G} \sim \mathrm{H}_{2} \mathrm{O}$ mutant shows only marginal activity detectable above the background (Table 2 and Table S5 for complete raw data). Coordination of Imid as a His mimic induces an activity of $67 \mathrm{U} \mathrm{mg} \mathrm{m}^{-1}$, corresponding to a 12 -fold reduction in activity with respect to the WT. These data are in agreement with previous investigations reported by Canters and co-workers who observed a 10 -fold reduction in activity upon rescuing the $\mathrm{H} 145 \mathrm{G}$ mutant with imidazole. ${ }^{[23]}$ In contrast, C-coordination of the NHC ligand increases the catalytic activity to $111 \mathrm{U} \mathrm{mg}^{-1}$, a nearly $100 \%$ enhancement when compared to H145G Imid. Due to the suboptimal occupancy achieved with both exogenous ligands, the measured activities are likely an underestimation of the full catalytic capacity of the rescued mutants, which when taking into account even the moderate contribution of the $\mathrm{H} 145 \mathrm{G} \sim \mathrm{H}_{2} \mathrm{O}$ activity, would even further enhance the effect of the NHC ligand.

Table 2. Catalytic nitrite reduction activity for NiR wild-type and H145G mutants containing Imid and NHC as exogenous ligands. 


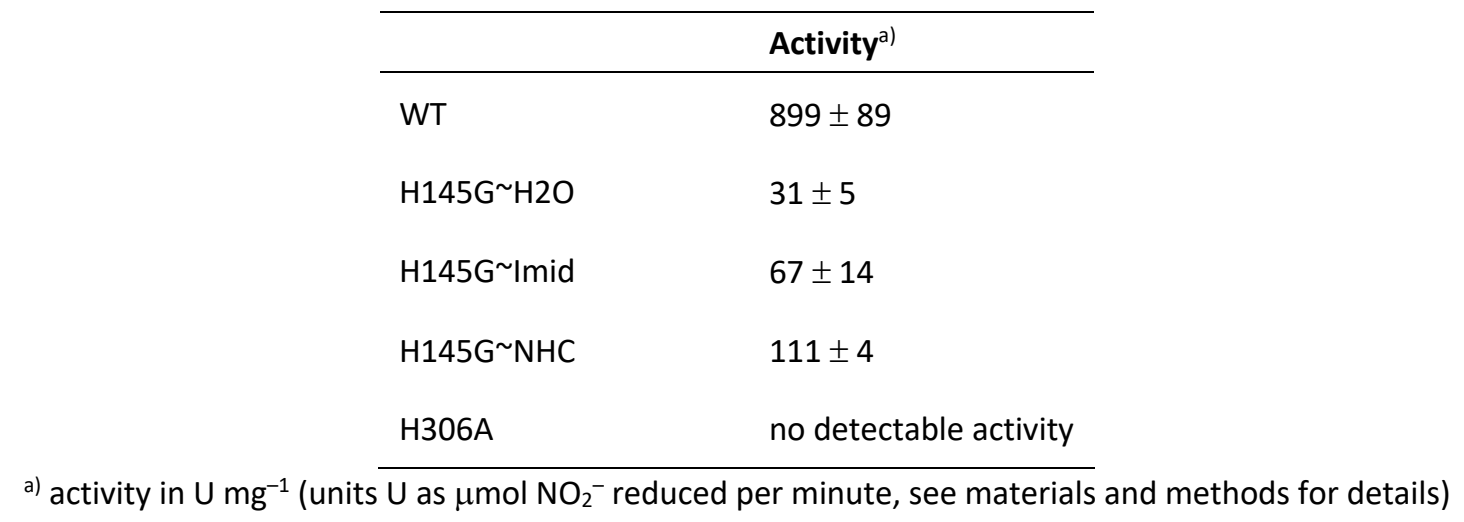

The significant enhancement of catalytic activities of the NHC mutant compared to the Imid analogue tentatively suggest that changing the coordination mode of the $\mathrm{N}$-heterocycle induces a shift in the rate limiting step, which is associated with the electron transfer process, i.e. the prime function of the peripheral T1 copper site. In the NiR WT, reversible oxidation and reduction of the T1 site is fast and promotes the shuttling of electrons from the sacrificial donor (Azu in the natural system, methyl viologen in the assay) to the catalytic T2 site. ${ }^{[20]}$ With imidazole as a static N-bound ligand in the H145G Imid variant, the T1 site is stabilized in its high-valent $\mathrm{Cu}(\mathrm{II})$ state. Assuming single electron transfer process to be generally fast in these enzymes (cf WT activity), this enhanced stabilization of the $\mathrm{Cu}(\mathrm{II})$ state limits electron transfer from the sacrificial donor to the T1 site and concomitant metal reduction to $\mathrm{Cu}(\mathrm{I})$. This step is substantially facilitated in the $\mathrm{H} 145 \mathrm{G} \sim \mathrm{NHC}$ mutant, since the softer carbene binding favors metal reduction to the lower-valent $\mathrm{Cu}(\mathrm{I})$ state ( $c f$ bleaching experiments and electrochemistry of Azu-NHC mutant). ${ }^{[31]}$ While static carbene bonding stabilizes the $\mathrm{Cu}(\mathrm{I})$ state and therefore increases the barrier for re-oxidation of the T1 copper center, the enhanced catalytic activity of the NHC mutant indicates that this penalty is considerably lower than that of the reduction in the NMI variant (Figure 5). This higher barrier rationalizes the lower activity of the NHC mutant compared to the WT. Such a mechanistic model therefore suggests that static ligand bonding is favoring the T1 center either as high-valent $\mathrm{Cu}(\mathrm{II})$ ( $\mathrm{N}$-bonding of Imid) or as low-valent $\mathrm{Cu}(\mathrm{I})$ (Cbonding of $\mathrm{NHC}$ ), which inherently slows down the catalytic rate. 


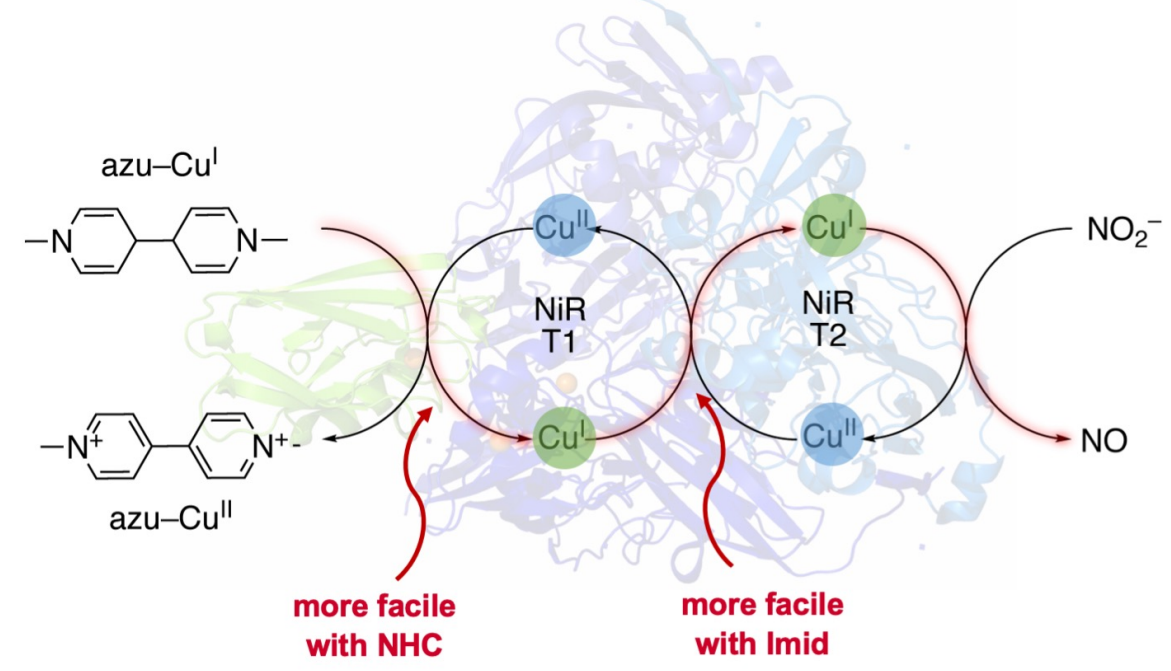

Figure 6. Schematic mode of action of NiR indicating the steps proposed to be promoted for the $\mathrm{H} 145 \mathrm{G} \sim \mathrm{NHC}$ variant (purple arrow) vs the $\mathrm{N}$-bound $\mathrm{H} 145^{\sim}$ Imid mutant (green arrow).

\section{Conclusion}

Here we have successfully introduced an exogeneous NHC ligand to the NiR H145G mutant, which reconstitutes the spectroscopic properties of the NiR WT. This new NiR H145G NHC variant constitutes a rare example of a blue copper protein containing a carbene ligand. Bleaching of the diagnostic green color has been demonstrated to occur due to reduction of the copper center from $\mathrm{Cu}(\mathrm{II})$ to $\mathrm{Cu}(\mathrm{I})$. This reactivity pattern is not observed with $\mathrm{N}$-bound ligands such as His or imidazole, and suggests stabilization of low-valent $\mathrm{Cu}(\mathrm{I})$ by the NHC ligand, in good agreement with earlier electrochemical analysis of the Azu mutants showing easier reduction of $\mathrm{Azu}-\mathrm{Cu}(\mathrm{NHC})$ than $\mathrm{Azu}-$ $\mathrm{Cu}(\mathrm{NMI})$. This propensity to stabilize low oxidation state $\mathrm{Cu}(\mathrm{I})$ also has a direct impact on the catalytic performance of $\mathrm{NiR}$ in nitrite reduction, as the rate limiting step is proposed to be shifted to reoxidation of the T1 copper site while electron transfer to the catalytic $\mathrm{Cu}$ center is fast and results in twice as high catalytic activity as compared to the imidazole-reconstituted variant. The benefit of carbene bonding would be greatly exploited if it were possible to engineer an amino acid with a carbene precursor side chain and devise a tRNA/aaRS system for genetic incorporation via AMBER stop-codon suppression $^{[36]}$ of this non-natural ligand in metalloenzyme active sites. Moreover, the 
fact that neither $\mathrm{C}$-bonding nor $\mathrm{N}$-bonding impart the activity of the WT, yet shift the rate limiting step, deserves further consideration and opens up the possibility that His could undergo transient tautomerism in metalloenzyme active sites during the catalytic cycle. This alternative C-bonding may be established by reversible wagging of $\mathrm{His}$ to induce tautomerization between the $\mathrm{C}$ - and $\mathrm{N}$-bonding mode. Such tautomerization may be particularly relevant for redox-active metallo-enzymes containing a His ligand bound to the metal center such as laccase or tyrosinase.

\section{Acknowledgements}

We thank Prof. Frank Neese (Max-Planck Institut, Mülheim an der Ruhr, Germany) for fruitful discussions. We thank the European Research Council through a Starting Grant (StG 208561) and a Consolidator grant (CoG 615653), the SELF program of Universität Bern (Switzerland), and the ICF scheme of the University of Nottingham (UK) for generous financial support.

Competing financial interests: The authors declare no competing financial interests.

Key words: metalloenzymes; histidine; N-heterocyclic carbene; ligand bonding mode; nitrite reductase.

\section{References}

[1] D. J. Nelson, S. P. Nolan, Chem. Soc. Rev. 2013, 42, 6723-53.

[2] M. N. Hopkinson, C. Richter, M. Schedler, F. Glorius, Nature 2014, 510, 485-496.

[3] E. Peris, Chem. Rev. 2018, 118, 9988-10031.

[4] D. Meyer, P. Neumann, R. Ficner, K. Tittmann, Nat. Chem. Biol. 2013, 9, 488-490.

[5] C. Mayer, D. G. Gillingham, T. R. Ward, D. Hilvert, Chem. Commun. 2011, 47, 12068-12070.

[6] M. Jeschek, R. Reuter, T. Heinisch, C. Trindler, J. Klehr, S. Panke, T. R. Ward, Nature 2016, 537, 661-665.

[7] M. Basauri-Molina, C. F. Riemersma, M. A. Würdemann, H. Kleijn, R. J. M. Klein Gebbink, Chem. Commun. 2015, 51, 6792-6795.

[8] R. D. Lewis, M. Garcia-Borràs, M. J. Chalkley, A. R. Buller, K. N. Houk, S. B. J. Kan, F. H. Arnold, Proc. Natl. Acad. Sci. 2018, 201807027.

[9] T. Hayashi, M. Tinzl, T. Mori, U. Krengel, J. Proppe, J. Soetbeer, D. Klose, G. Jeschke, M. Reiher, D. Hilvert, Nat. Catal. 2018, 1, 578-584. 
[10] M. Planchestainer, N. Segaud, M. Shanmugam, J. McMaster, F. Paradisi, M. Albrecht, Angew. Chemie - Int. Ed. 2018, 57, 10677-10682.

[11] S. J. Lippard, J. M. Berg, Principles of Bioinorganic Chemistry, University Science Books, Mill Valley, CA, 1994.

[12] A. Sigel, H. Sigel, R. K. O. Sigel, Metal lons in Life Science, Royal Society Of Chemistry, London, UK, 2009.

[13] J. P. Collman, R. Boulatov, C. J. Sunderland, L. Fu, Chem. Rev. 2004, 104, 561-588.

[14] E. I. Solomon, R. K. Szilagyi, S. DeBeer George, L. Basumallick, Chem. Rev. 2004, 104, 419-458.

[15] P. Wittung-Stafshede, M. G. Hill, E. Gomez, A. J. Di Bilio, B. G. Karlsson, J. Leckner, J. R. Winkler, H. B. Gray, B. G. Malmström, J. Biol. Inorg. Chem. 1998, 3, 367-370.

[16] T. Kohzuma, S. Shidara, S. Suzuki, Bull. Chem. Soc. Jpn. 1994, 67, 138-143.

[17] M. D. Vlasie, R. Fernández-Busnadiego, M. Prudêncio, M. Ubbink, J. Mol. Biol. 2008, 375, $1405-1415$.

[18] P. Comba, W. Schiek, Coord. Chem. Rev. 2003, 238-239, 21-29.

[19] J. Stanek, A. Hoffmann, S. Herres-Pawlis, Coord. Chem. Rev. 2018, 365, 103-121.

[20] Y. Li, M. Hodak, J. Bernholc, Biochemistry 2015, 54, 1233-1242.

[21] S. Horrell, D. Kekilli, K. Sen, R. L. Owen, F. S. N. Dworkowski, S. V. Antonyuk, T. W. Keal, C. W. Yong, R. R. Eady, S. S. Hasnain, et al., IUCrJ 2018, 5, 283-292.

[22] S. Horrell, S. V. Antonyuk, R. R. Eady, S. S. Hasnain, M. A. Hough, R. W. Strange, IUCrJ 2016, 3, $271-281$.

[23] H. J. Wijma, M. J. Boulanger, A. Molon, M. Fittipaldi, M. Huber, M. E. P. Murphy, M. P. Verbeet, G. W. Canters, Biochemistry 2003, 42, 4075-4083.

[24] A. Monney, M. Albrecht, Chem. Commun. 2012, 48, 10960-10962.

[25] H. Nar, a. Messerschmidt, R. Huber, M. Van De Kamp, G. W. Canters, FEBS Lett. 1992, 306, 119-124.

[26] H. J. Wijma, I. MacPherson, O. Farver, E. I. Tocheva, I. Pecht, M. P. Verbeet, M. E. P. Murphy, G. W. Canters, J. Am. Chem. Soc. 2007, 129, 519-525.

[27] A. Stirpe, L. Sportelli, H. Wijma, M. P. Verbeet, R. Guzzi, Eur. Biophys. J. 2007, 36, 805-813.

[28] T. Den Blaauwen, G. W. Canters, J. Am. Chem. Soc. 1991, 113, 5050-5052.

[29] M. Van de Kamp, F. C. Hali, N. Rosato, A. F. Agro, G. W. Canters, Biochim. Biophys. Acta - Bioenerg. 1990, 1019, 283292.

[30] T. Den Blaauwen, G. W. Canters, J. Am. Chem. Soc. 1993, 115, 1121-1129.

[31] Á. Vivancos, C. Segarra, M. Albrecht, Chem. Rev. 2018, acs.chemrev.8b00148.

[32] N. A. Sieracki, S. Tian, R. G. Hadt, J. L. Zhang, J. S. Woertink, M. J. Nilges, F. Sun, E. I. Solomon, Y. Lu, Proc. Natl. Acad. Sci. U. S. A. 2014, 111, 924-929.

[33] C. Hammann, G. van Pouderoyen, H. Nar, F.-X. G. Rüth, A. Messerschmidt, R. Huber, T. den Blaauwen, G. W. Canters, J. Mol. Biol. 1997, 266, 357-365.

[34] E. Wyer, G. Gucciardo, V. Leigh, H. Müller-Bunz, M. Albrecht, J. Organomet. Chem. 2011, 696, $2882-2885$.

[35] L. Krzeminski, S. Cronin, L. Ndamba, G. W. Canters, T. J. Aartsma, S. D. Evans, L. J. C. Jeuken, J. Phys. Chem. B 2011, $115,12607-12614$.

[36] T. S. Young, P. G. Schultz, J. Biol. Chem. 2010, 285, 11039-11044. 